\title{
Minireview
}

\section{Strategies for Improving Responses to DNA Vaccines}

\author{
J. S. Boyle, ${ }^{1}$ I. G. Barr, ${ }^{2}$ and A. M. Lew ${ }^{1}$
}

${ }^{1}$ Co-operative Research Centre for Vaccine Technology, The Walter and

Eliza Hall Institute of Medical Research, Melbourne, Australia, 3050

${ }^{2}$ Research and Development Division, CSL Ltd., Parkville, Australia, 3052

\section{Introduction}

A DNA vaccine consists of a plasmid that allows expression of an antigen encoded by an inserted gene or cDNA in mammalian cells as directed by a strong promoter element, usually of viral origin (i.e., human cytomegalovirus, rous sarcoma virus). The first report that intramuscular (i.m.) injection of plasmid DNA could be used as a mode for vaccination sparked worldwide interest (1). It has also been shown that other routes of DNA delivery such as intradermal (i.d.) injection (2) or facilitated transfer such as propulsion of DNA coated particles with a gene gun (3) would also result in expression of encoded antigens and induction of immune responses. DNA vaccines could be considered similar to viruses except that they are nonreplicating and lack the pathological consequences. Indeed, the immune response to a DNA vaccine mimics that of a viral antigen after infection $(4,5)$ with both cytotoxic T lymphocyte (CTL) and antibody (Ab) responses elicited.

The mechanism by which the DNA injected in saline enters the cells is unknown except that it is inefficient (compared to viral delivery) and may vary depending upon delivery method, as would the target cell population. I.m. injection results in low-level transfection of myocytes (6) whereas i.d. injection or gene gun delivery may directly transfect antigen-presenting cells (APCs) $(2,7)$. There is one report of a very low level of macrophages that are transfected after i.m. injection (8). The mechanism of priming with the

Address correspondence and reprint requests to: Dr. Andrew M. Lew, Co-operative Research Centre for Vaccine Technology, The Walter and Eliza Hall Institute of Medical Research, P.O. Box RMH, Melbourne, Australia, 3050. Phone: 6139345 2555; Fax: 6139347 0852; E-mail: lew@wehi.edu.au different routes and exactly what cell types are involved have not been fully resolved for DNA immunization. Perhaps this is not surprising, as the majority of the research to date has concentrated on protection studies. This review will focus on the application of DNA vaccines and present a brief overview of the current state of the field and how researchers are overcoming the hurdles in their efforts to realize the clinical potential of DNA vaccines. In particular, emphasis has been placed upon research into methods that could be used to increase the potency of DNA vaccines.

\section{The Potential}

Much of the interest in DNA vaccines is largely due to what this facile, generic, and enabling technology offers. A list of the potential advantages and problems facing DNA vaccines is given in Table l. Some of these advantages are due to properties inherent to DNA. The physiochemical stability and homogeneity of DNA should avail for easily produced inexpensive vaccines. Because DNA immunization is a form of in vivo transfection, the production of antigen will be in mammalian cells and antigens normally produced in such cells (e.g., viral protein) will thus be in native conformation. As in a viral infection, a broad (both cellular and humoral) immune response is induced. DNA vaccines may also be combined relatively easily, a feature that can be difficult for conventional recombinant vaccines in which protein solubility conditions differ. Overall, the advantages of DNA vaccines warrant the large research effort and expenditure, but the problems facing the technology will be difficult to overcome. In particular, there is a possibility 
Table 1. The potential of DNA vaccines

Advantages

Noninfectious/nonreplicating

Proteins produced in native conformation, particularly viral proteins

Physiochemical homogeneity; possibly easier to incorporate several components in a single vaccine

Simplified production

Physiochemical stability

Induction of CTLs

Utility of molecular biology

\section{Some Problems}

Potential for insertion into the genome

Potency of immune responses, particularly in larger animals and humans

Autoimmunity that integration of the DNA vaccine plasmid into the host cell genome either randomly or by homologous recombination could lead to insertional mutagenesis. Such an event might result in activation of oncogenes, inactivation of tumor suppressor genes, or chromosomal instability. Thus far, experimental evidence has shown that the risk of integration is extremely low $(9,10)$, but this perceived risk may still restrict the application of DNA vaccines. Perhaps the greatest problem facing DNA vaccines will be efficacy in large animals and the transition to humans; efforts are now being concentrated on improving the immunogenicity and efficacy of DNA vaccines (discussed later).

\section{Role for DNA Vaccines in Research and Development}

The eventual clinical role for DNA vaccines is currently being evaluated, but the success of DNA immunization in mouse models in particular has almost certainly entrenched it within research and development programs. With DNA vaccines, the foreign protein (selected from the organism e.g., hepatitis B surface antigen) is synthesized by the host's own cells and both an $\mathrm{Ab}$ and $\mathrm{T}$ cell response can be initiated. Thus, researchers have a useful tool in which to study immune responses to antigens in the absence of adjuvant and contaminating proteins and, for example, could study immunity relevant to viral infection without the influence of viral biology (i.e., nonlytic, noninfectious). The absence of an antigen purification regimen could also potentially lead to easy production of monoclonal $\mathrm{Ab}$ for research and development programs $(11,12)$.
$\mathrm{Ab}$ reagents produced by DNA immunization could have the benefit of recognizing native or conformational determinants. This application has probably not been put to its full potential perhaps because of the need for a source of native antigen for screening. In this regard, it is worth considering the concept of original antigenic sin in immunology whereby the $\mathrm{Ab}$ response to a secondary immunization of a related antigen leads to primarily Abs of the primary specificity. Therefore, DNA immunization followed by bacterially derived recombinant antigen may reap the benefit of both-i.e., high $\mathrm{Ab}$ levels with native specificity.

For future vaccine candidate identification and development, DNA vaccines have the potential to simplify dramatically the production, purification, storage, and combination of different vaccines (Table 1 ). This could save enormous amounts of time and investment in evaluating vaccine candidate antigens in the laboratory and in producing vaccines (Fig. 1), as efficacy testing could commence in the order of weeks as opposed to months or even years for some recombinant proteins. An extreme example is expression library immunization (13) whereby the entire cDNA population or genome is used as a vaccine or as a starting point from which candidates could be identified via a process of elimination. This is more likely to be successful for screening pathogens with relatively small genomes. When compared to more conventional systems, the timesaving for large-scale evaluation of antigens is at least, in theory, enormous (Fig. 1). A word of caution may be appropriate here for the testing of DNA vaccine candidates in research. The presence of endotoxin can produce misleading results (14) and it is essential to elim- 


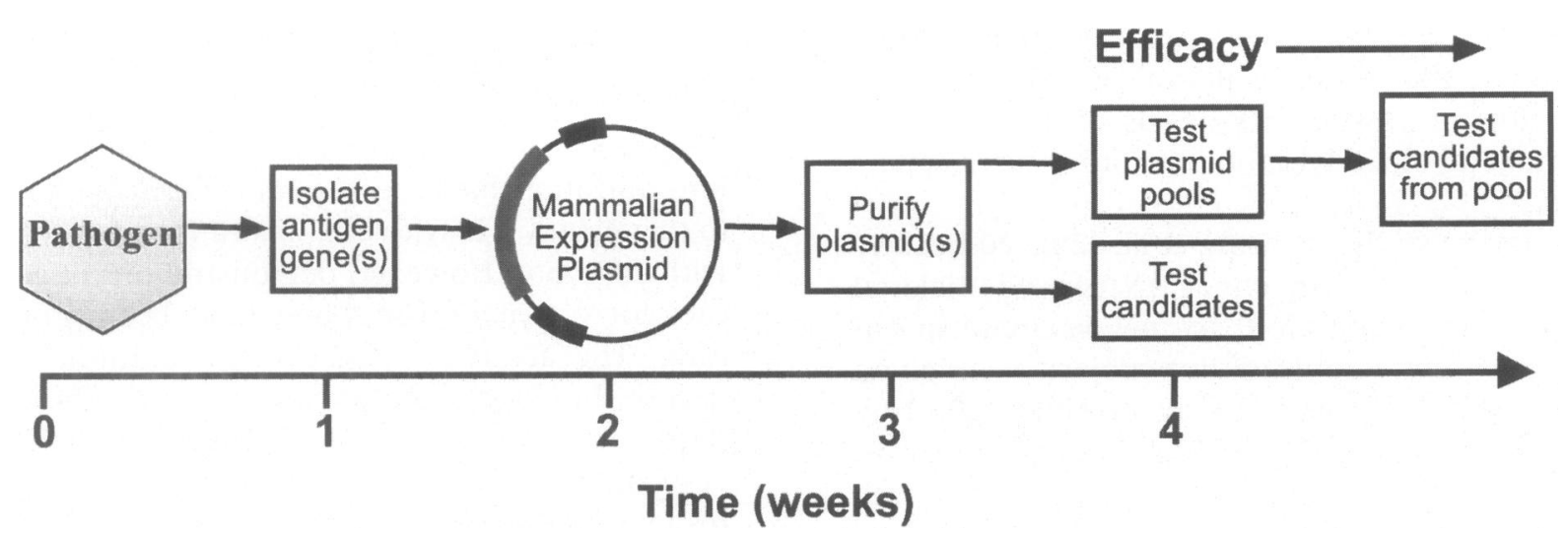

Fig. 1. Process of evaluating DNA vaccine candidate antigens.

inate it during DNA isolation, e.g., using Triton X-114 phase extraction, a QIAGEN endofree plasmid purification kit, or double $\mathrm{CsCl}$ gradient centrifugation.

\section{Potential Clinical Targets for DNA Vaccines}

While traditional vaccine development using whole inactivated organisms or, more recently, subunit vaccines have produced a substantial number of human and animal vaccines with many more under development, there are still a number of diseases in which DNA vaccines may be applied. It is unlikely, even if they are very successful, that DNA vaccines will replace existing vaccines such as tetanus, diphtheria, measles, mumps, rubella, influenza, etc. because of the market acceptance, low cost, proven effectiveness, and low incidence of side effects of these existing vaccines. DNA vaccines are also unlikely to replace some of the newer vaccines such as hepatitis $B$, hepatitis $A$, and varicella for similar reasons or the newer pediatric combination vaccines that now contain several antigens. Nonprotein vaccines, such as hemophilus influenza B and multivalent pneumococcal vaccines, that are based on polysaccharide antigens also cannot be directly substituted by DNA vaccines.

There are many infectious diseases for which there is no vaccine available. A short list of major human pathogens would include HIV, Plasmodium spp., hepatitis C, Mycobacterium tuberculosis, human papilloma virus, rotavirus, shigella, Epstein-Barr virus (EBV), Leishmania spp., etc. Interestingly, in animal models of many of these diseases, DNA vaccines have proven remarkably effective. While much of the initial work on DNA vaccines has been targeted at infectious disease models, they are also being trialed in cancer models in animals (15-17) and in human trials for lymphoma (Professor F. Stevenson, Southampton University, Southampton, U.K.) and melanoma (Dr S. Rosenberg, National Cancer Institute, Bethesda, MD) and may also be used in the future in the treatment of autoimmunity and allergies.

\section{Current Human Clinical Trials}

At this stage, only a few groups have reported results with DNA vaccines in phase I human trials. Two of these studies investigated the responses to HIV-1-encoded proteins $(18,19)$, while another investigated the CSP protein of Plasmodium falciparum (20). The Calarota study (18) involved three groups of three subjects (HIV-l infected but asymptomatic) who were each given three doses of $100 \mu \mathrm{g}$ of plasmid encoding the cDNA for either nef, rev, or tat HIV-1 genes intramuscularly in distilled water. They reported that DNA vaccination induced detectable memory cells in all subjects and specific CTLs in eight out of nine subjects that were above preimmunization levels. However, three of these responses were transient. There were no apparent side effects reported.

In the MacGregor study (19), three groups of five subjects (HIV-1 infected but asymptomatic) were given 30,100 , or $300 \mu \mathrm{g}$ of plasmid DNA encoding modified env and rev HIV- $\mathrm{l}_{\mathrm{MN}}$ genes intramuscularly. No local or systemic reactions or laboratory abnormalities were seen and no anti-DNA antibodies were detected. Slight increases in antibody titers to recombinant HIV- 
$l_{M N}$ gp120 and CTL were seen in some patients at the higher doses of plasmid DNA but no reduction in plasma HIV levels or CD4 or CD8 peripheral blood lymphocyte counts were apparent.

In the study by Wang et al. (20), 20 healthy volunteers were given a DNA-based malarial vaccine encoding the $P$. falciparum circumsporozoite protein. Groups of five subjects were given three doses intramuscularly of either 20, 100, 500 , or $2500 \mu \mathrm{g}$ of DNA at four weekly intervals. The vaccine was reported to be safe and well tolerated with the majority of subjects developing CTL responses in a dose-related manner. While the immunological responses in the published trials to date appear to have been modest, their initial focus has been on establishing the safety of DNA vaccine, and many subjects have been given low amounts of DNA, similar to what many experimenters have given to mice. In addition, the subjects in the HIV trials, while being asymptomatic, were HIV-l infected, and this may have compromised their responses to DNA vaccination. The release of further human data from trials including those that have examined hepatitis B and influenza DNA vaccines is keenly awaited. It seems probable that strategies may need to be modified to achieve satisfactory human efficacy with DNA vaccines. These include modifications such as enhancing the uptake of DNA, targeting of the encoded proteins, or use of a prime-boost (DNA vaccine given in the initial dose followed by recombinant protein or virus).

\section{Hurdles for DNA Vaccines}

As mentioned earlier, gaining evidence to allay the safety concerns (Table 1) will be paramount for the progression of DNA vaccines. The clinical trials underway and the few that have been completed thus far should aid in these efforts. Nonetheless, the transition of DNA vaccines from the mouse to larger animals and humans has not been easy. Efficacy remains an issue. Protection data on naive humans need to be obtained. Furthermore, work in murine models examining prime boost strategies demonstrates that the least effective method is to prime with virus and "boost" with DNA, whereas the reverse may be optimal (21). Nonetheless, there does appear to be scope for improving the immune response and efficacy achievable with DNA vaccines.

\section{Increasing Potency of DNA Vaccines}

In general, immune responses following DNA immunization have been poor when compared to that achievable with administration of antigen with adjuvant. However, demonstrations of efficacy for vaccination purposes have been promising. The ability to enhance the immune response should lead to increased vaccine efficacy. Three of the many systems currently under investigation to increase the potency of the $A b$ response to a DNA vaccine are illustrated for, as an example, i.m. injection of DNA in Figure 2.

The addition of immunostimulatory sequences (CpG motifs) resulted in elevated $\mathrm{Ab}$ responses to DNA-encoded antigens following i.d. DNA injection $(22,23)$. Another strategy was to use cytokines to influence the immune response. It was shown that when antigen-encoding DNA was co-injected with granulocyte-macrophage colony stimulating factor (GM-CSF), an enhanced $\mathrm{Ab}$ response was achieved, whereas the same strategy using interferon $\gamma$ (INF- $\gamma$ ) decreased the $\mathrm{Ab}$ response (24). Others have shown very modest enhancement of $\mathrm{Ab}$ responses to co-administered antigen due to GMCSF $(0.1$ OD change at a serum dilution of $1: 100)$ with no effect on the cellular response (25). Furthermore, GM-CSF co-injection led to increases in seroconversion rates (percentage of mice testing positive for $\mathrm{Ab}$ responses) but not the magnitude of the $A b$ response (26). Dramatic increases in antigen-specific CTL activity have been shown with co-administration of genes encoding interleukin (IL)-12 (25) or IL-2 (26). The enhancement achieved varies among systems and may therefore be somewhat dependent on the antigen. The mechanism by which cytokine gene transfer could augment antigen-specific immunity is unclear. Perhaps when the mechanisms in the immunobiology of DNA immunization are unraveled, approaches based on such rationale rather than empiricism will prove important.

An alternative to cytokine gene transfer in which cells of the immune system are the targets for activation is to modify the transfected cell. Myocytes lack co-stimulation and do not prime a CTL response directly after i.m. DNA injection. Instead, priming is achieved by nontransfected bone marrow-derived cells via a cross-presentation mechanism (27-29). Co-injection of genes encoding the B7 co-stimulator molecules has been touted as a method to overcome this problem. One group demonstrated an elevation in 


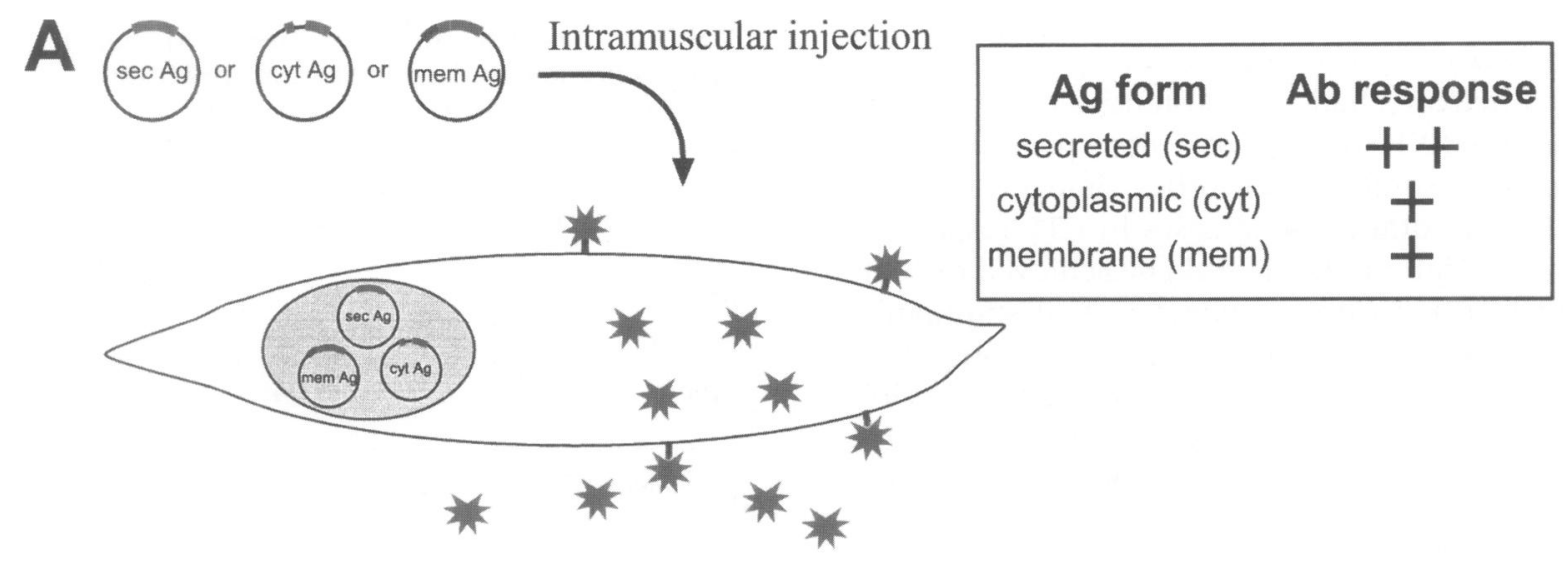

B

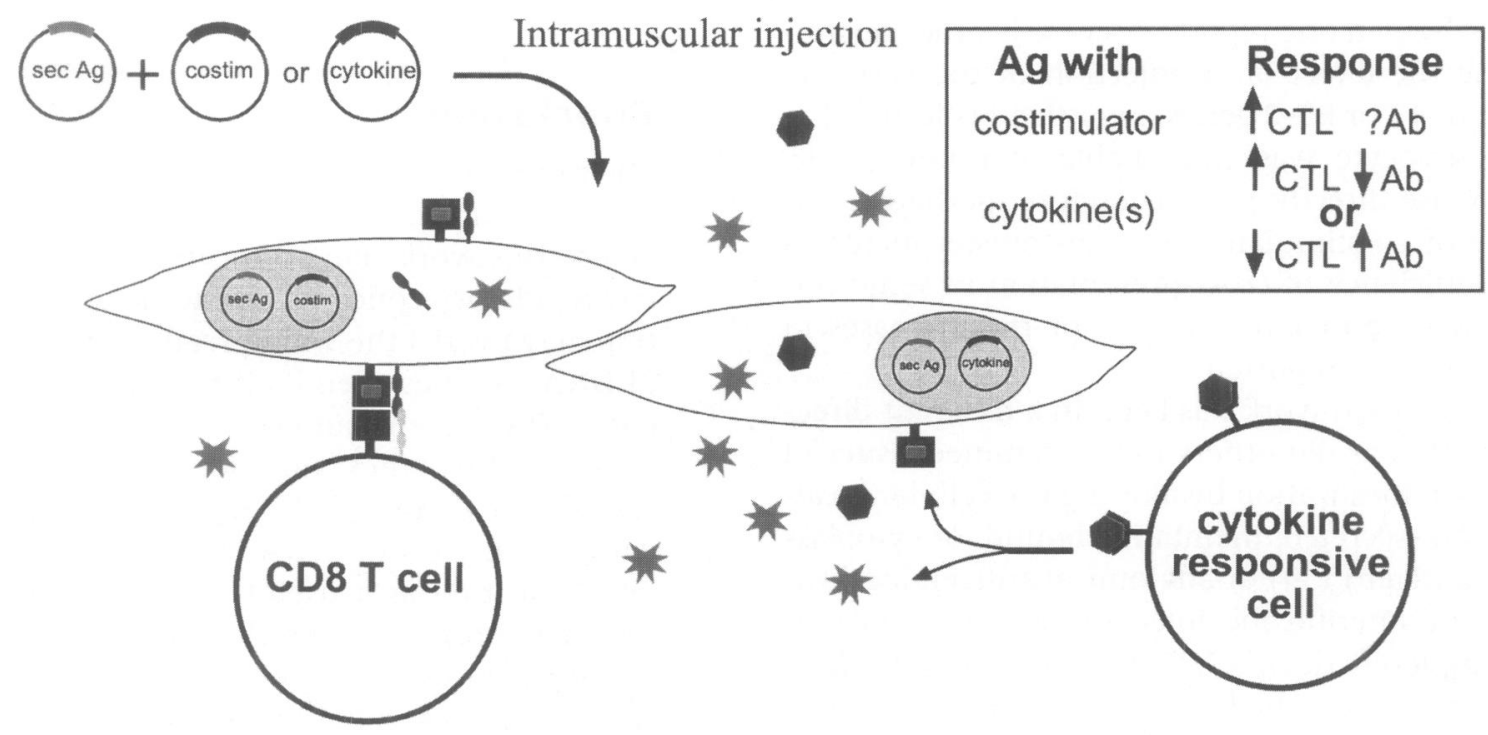

C

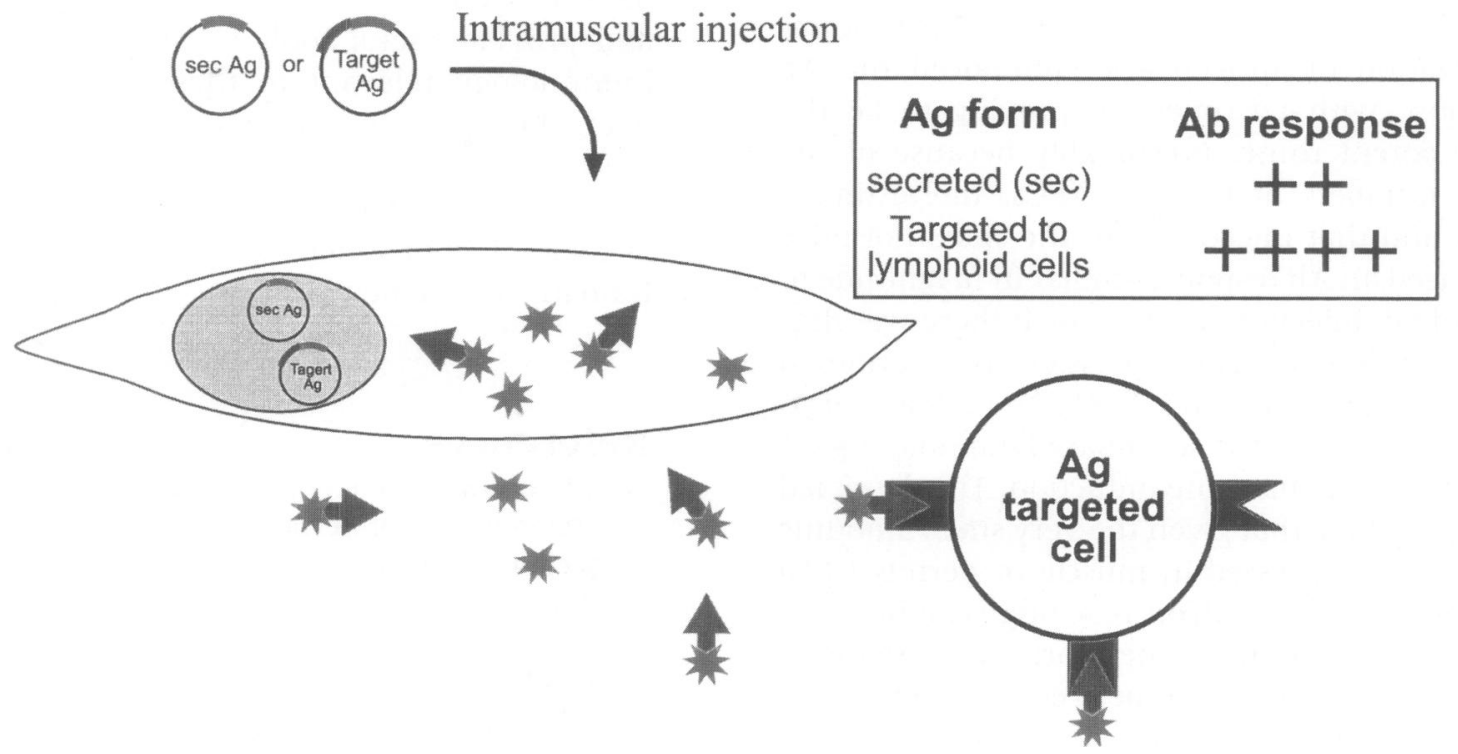

Fig. 2. Three systems under investigation to increase potency of Ab response to a DNA vaccine. (A) Secreted, cytoplasmic, or membrane antigens (Ag) are injected i.m. (B) Secreted antigen plus costimulator or cytokine(s) are injected i.m. (C) Secreted antigen or antigen targeted to lymphoid cells is secreted. 
antigen-specific $\mathrm{Ab}$ responses after $\mathrm{B} 7-\mathrm{l}$ co-delivery (30). In contrast, another group showed B7 co-delivery had no substantial effect on $\mathrm{Ab}$ responses (0.1 OD shift at 8 days post-immunization but similar at all other times), but suggested that dramatic increases in CTL responses with B7-2 but not B7-1 could be achieved (31). It is unclear as to how provision of co-stimulator molecules could enhance Ab response when the myocyte lacks class II expression and therefore cannot present antigen to these helper $\mathrm{T}$ cells. In the case of CTL responses, it could be that costimulator provision provides the myocyte with the capability to prime a CTL response directly. However, in one report (discussed earlier) examining the effect of co-injection of the GM-CSF and IL-12 or B7-2 genes, investigators found that the myocyte was still unable to prime a CTL response directly (32). Therefore, perhaps cytokine or co-stimulator gene co-transfer increases the efficiency of cross-presentation in some unknown manner to account for the increases in CTL activity reported.

Our own work has been in a different direction. We, as did others (33), examined issues of antigen localization by altering the cellular localization (secreted, membrane bound, or cytoplasmic antigen) (34) or anatomical antigen localization by altering the injection site [i.m., i.d., or intrasplenic (i.s.); $(2,35,36)]$. Cellular localization of antigen had profound effects on immunity and it was shown that secreted antigen produced the highest $A b$ response. Anatomical localization of antigen also influenced the $A b$ response with i.d. injection proving to be the most potent route, presumably because of the large numbers of resident APCs. Interestingly, we found that injection into the spleen of mice produced an $\mathrm{Ab}$ response similar in magnitude to that of i.d. injection, even though there was dramatically less protein expression as determined using luciferase reporter (Table 2). Perhaps this is not surprising, as the secondary lymphoid organs are the sites of immune induction. This work led us to conclude that given the very small amounts of antigen expressed in muscle or dermis $(<10$ ng), the level of antigen reaching lymphoid organs may be limiting. Therefore, we designed a soluble fusion molecule between antigen and a ligand whose receptors are associated with lymphoid organs such that the immune response to the antigen could be dramatically increased (37). Thus, different methods of varying antigen localization could be used to improve and influence the form of the immune response.
Table 2. Magnitude of immune response in spleen is maximal even though protein expression levels are very low

\begin{tabular}{lcc}
\hline Site of Injection & $\begin{array}{c}\text { Protein Expression }^{a} \\
\text { (ng) }\end{array}$ & Ab Levels \\
\hline Muscle & 10 & ++ \\
Dermis & 9 & +++ \\
Spleen & 0.04 & +++ \\
& & \\
\hline
\end{tabular}

${ }^{a}$ As determined with a luciferase reporter.

\section{Conclusions}

The numerous potential advantages of DNA vaccination warrant further work, but the hurdles before this work remain formidable. In particular, regulatory concerns due to possible integration may restrict the widespread prophylactic use of DNA vaccines even if efficacy issues are overcome. The current human clinical trials may well answer some of these questions and we hope will suggest the continual development of DNA vaccines. In any event, DNA immunization has established itself as a useful tool for the research scientist and vaccine development program. The stunning successes in murine models demonstrate that DNA could be used to identify candidate antigens for more conventional vaccines and provide useful tools to understanding the immunology relevant to these models. Moreover, these models may eventually unravel the mechanisms of DNA uptake and immune induction. These mechanisms may then be manipulated for higher efficiency and thus efficacy in humans be augmented.

\section{References}

1. Ulmer JB, Donnelly JJ, Parker SE, et al. (1993) Heterologous protection against influenza by injection of DNA encoding a viral protein. Science 259: 1745-1749.

2. Raz E, Carson DA, Parker SE, et al. (1994) Intradermal gene immunization: the possible role of DNA uptake in the induction of cellular immunity to viruses. Proc. Natl. Acad. Sci. U.S.A. 91: 95199523.

3. Tang DC, De Vit M, Johnston SA (1992) Genetic immunization is a simple method for eliciting an immune response. Nature 356: 152-154.

4. Mor G, Klinman DM, Shapiro S, et al. (1995) 
Complexity of the cytokine and antibody response elicited by immunizing mice with Plasmodium yoelii circumsporozoite protein plasmid DNA. J. Immunol. 155: 2039-2046.

5. Michel ML, Davis HL, Schleef M, Mancini M, Tiollais P, Whalen RG (1995) DNA-mediated immunization to the hepatitis $B$ surface antigen in mice: aspects of the humoral response mimic hepatitis B viral infection in humans. Proc. Natl. Acad. Sci. U.S.A. 92: 5307-5311.

6. Wolff JA, Malone RW, Williams P, et al. (1990) Direct gene transfer into mouse muscle in vivo. Science 247: 1465-1468.

7. Condon C, Watkins SC, Celluzzi CM, Thompson K, Falo LD Jr. (1996) DNA-based immunization by in vivo transfection of dendritic cells. Nature Med. 2: 1122-1128.

8. Chattergoon MA, Robinson TM, Boyer JD, Weiner DB (1998) Specific immune induction following DNA-based immunization through in vivo transfection and activation of macrophages/antigenpresenting cells. J. Immunol. 160: 5707-5718.

9. Wolff JA, Ludtke JJ, Acsadi G, Williams P, Jani A. (1992) Long-term persistence of plasmid DNA and foreign gene expression in mouse muscle. Hum. Mol. Genet. 1: 363-369.

10. Nichols WW, Ledwith BJ, Manam SV, Troilo PJ. (1995) Potential DNA vaccine integration into host cell genome. Ann. N. Y. Acad. Sci. 772: 30-39.

11. Ulivieri C, Burroni D, Telford JL, Baldari CT. (1996) Generation of a monoclonal antibody to a defined portion of the Helicobacter pylori vacuolating cytotoxin by DNA immunization. J. Biotechnol. 51: 191-194.

12. Barry MA, Barry ME, Johnston SA (1994) Production of monoclonal antibodies by genetic immunization. Biotechniques 16: 616-618.

13. Barry MA, Lai WC, Johnston SA (1995) Protection against mycoplasma infection using expression-library immunization. Nature 377: 632-635.

14. Boyle JS, Brady JL, Koniaras C, Lew AM. (1998) The inhibitory effect of lipopolysaccharide on the immune response after DNA immunization is route dependent. DNA Cell Biol. 17: 343-348.

15. Chen $\mathrm{Y}, \mathrm{Hu} \mathrm{D}$, Eling DJ, Robbins J, Kipps $\mathrm{TJ}$ (1998) DNA vaccines encoding full-length or truncated Neu induce protective immunity against Neu-expressing mammary tumors. Cancer Res. 58: 1965-1971.

16. Ciernik IF, Berzofsky JA, Carbone DP. (1996) Induction of cytotoxic $\mathrm{T}$ lymphocytes and antitumor immunity with DNA vaccines expressing single $\mathrm{T}$ cell epitopes. J. Immunol. 156: 2369-2375.

17. Iwasaki A, Barber BH. (1998) Induction by DNA immunization of a protective antitumor cytotoxic $\mathrm{T}$ lymphocyte response against a minimalepitope-expressing tumor. Cancer Immunol. Immunother. 45: 273-279.

18. Calarota S, Bratt G, Nordlund S, et al. (1998) Cellular cytotoxic response induced by DNA vac- cination in HIV-1-infected patients. Lancet 351: 1320-1325.

19. MacGregor RR, Boyer JD, Ugen KE, et al. (1998) First human trial of a DNA-based vaccine for treatment of human immunodeficiency virus type 1 infection: safety and host response. J. Infect. Dis. 178: $92-100$.

20. Wang R, Doolan DL, Le TP, et al. (1998) Induction of antigen-specific cytotoxic T lymphocytes in humans by a malaria DNA vaccine. Science 282: 476480.

21. Schneider J, Gilbert SC, Blanchard TJ, et al. (1998) Enhanced immunogenicity for CD8 $+\mathrm{T}$ cell induction and complete protective efficacy of malaria DNA vaccination by boosting with modified vaccinia virus Ankara. Nat. Med. 4: 397-402.

22. Sato $Y$, Roman $M$, Tighe $H$, et al. (1996) Immunostimulatory DNA sequences necessary for effective intradermal gene immunization. Science 273: 352-354.

23. Roman M, Martin-Orozco E, Goodman JS, et al. (1997) Immunostimulatory DNA sequences function as T helper-1-promoting adjuvants. Nat. Med. 3: 849-854.

24. Xiang Z, Ertl HC. (1995) Manipulation of the immune response to a plasmid-encoded viral antigen by coinoculation with plasmids expressing cytokines. Immunity 2: 129-135.

25. Kim JJ, Ayyavoo V, Bagarazzi ML, et al. (1997) In vivo engineering of a cellular immune response by coadministration of IL- 12 expression vector with a DNA immunogen. J. Immunol. 158: 816-826.

26. Geissler M, Gesien A, Tokushige K, Wands JR. (1997) Enhancement of cellular and humoral immune responses to hepatitis $C$ virus core protein using DNA-based vaccines augmented with cytokine-expressing plasmids. J. Immunol. 158: 12311237.

27. Corr M, Lee DJ, Carson DA, Tighe H. (1996) Gene vaccination with naked plasmid DNA: mechanism of CTL priming. J. Exp. Med. 184: 1555-1560.

28. Doe B, Selby M, Barnett S, Baenziger J, Walker CM. (1996) Induction of cytotoxic T lymphocytes by intramuscular immunization with plasmid DNA is facilitated by bone marrow derived cells. Proc. Natl. Acad. Sci. U.S.A. 93: 8578-8582.

29. Ulmer JB, Deck RR, Dewitt CM, Donnelly JJ, Liu MA (1996) Generation of MHC class I-restricted cytotoxic $\mathrm{T}$ lymphocytes by expression of a viral protein in muscle cells: antigen presentation by non-muscle cells. Immunology 89: 59-67.

30. Conry RM, Widera G, LoBuglio AF, et al. (1996) Selected strategies to augment polynucleotide immunization. Gene Ther. 3: 67-74.

31. Kim JJ, Bagarazzi ML, Trivedi N, et al. (1997) Engineering of in vivo immune responses to DNA immunization via codelivery of costimulator molecule genes. Nat. Biotech. 15: 641-646.

32. Iwasaki A, Torres CA, Ohashi PS, Robinson HL, Barber BH. (1997) The dominant role of bone 
marrow-derived cells in CTL induction following plasmid DNA immunization at different sites. J. Immunol. 159: 11-14.

33. Xiang ZQ, Spitalnik SL, Cheng J, Erikson J, Wojczyk B, Ertl HC. (1995) Immune responses to nucleic acid vaccines to rabies virus. Virology 209: 569-579.

34. Boyle J, Koniairas C, Lew A. (1997) Influence of cellular location of expressed antigen on the efficacy of DNA vaccination: cytotoxic T lymphocyte and antibody responses are suboptimal when antigen is cytoplasmic after intramuscular DNA immunization. Int. Immunol. 9: 1897-1906.
35. Boyle JS, Silva A, Brady JL, Lew AM. (1997) DNA immunization: induction of higher avidity antibody and effect of route on $\mathrm{T}$ cell cytotoxicity. Proc. Natl. Acad. Sci. U.S.A. 94: 14626-14631.

36. Gerloni M, Billetta R, Xiong S, Zanetti M. (1997) Somatic transgene immunization with DNA encoding an immunoglobulin heavy chain. DNA Cell Biol. 16: 611-625.

37. Boyle JS, Brady JL, Lew AM. (1998) Enhanced responses to a DNA vaccine encoding a fusion antigen that is directed to sites of immune induction. Nature 392: 408-411. 